\title{
Battlefield Acupuncture for Chronic Pain Management in Patients on Long-Term Opioid Therapy
}

\author{
Anna Denee Montgomery, BS, and Ronovan Ottenbacher, MD
}

\begin{abstract}
Objectives: Battlefield Acupuncture (BFA) is a unique auricular acupuncture procedure utilized by many Veterans Affairs Healthcare Administration facilities. Several previous studies have shown an immediate reduction in pain for up to 2 weeks post BFA. The long-term effects of BFA and its potential to decrease opioid use had yet to be analyzed. This study was conducted to analyze the effectiveness of BFA to decrease chronic pain immediately and 6 months after treatment and to decrease the number of opioids needed for management of chronic pain.

Materials and Methods: This was a retrospective cohort study comparing veterans who received BFA and were prescribed opioids for their chronic pain to veterans who did not receive BFA. The treatment group included 24 veterans who received BFA and had opioid contracts. The comparison group consisted of 23 randomly selected veterans who had opioid contracts but did not receive BFA. A numeric rating scale (NRS) was used to measure pain before and after treatment, as well as 3 months prior and 6 months post. The average morphine mg equivalents for opioids 3 months prior and 6 months post treatment were also compared. Differences between groups were statistically analyzed by an analysis of variance and a Student's $t$-test.

Results: Significant average decreases of 1.3 points on the NRS occurred in $66.1 \%$ immediately after the procedure. No significant decreases in pain were found. No significant changes of the average number of opioids over the 9 months analyzed were found.

Conclusions: BFA is effective for immediate pain reduction. Further research with a randomized controlled trial in a larger population is needed to assess BFA effects on chronic pain and opioid dependency.
\end{abstract}

Keywords: Battlefield Acupuncture, acupuncture, opioid, pain, pain management

\section{INTRODUCTION}

$\mathbf{P}$ AIN MANAGEMENT FOR Veterans is an ongoing challenge that is managed primarily through medications, with opioids being the most widely used to treat chronic pain. ${ }^{1}$ For the Veterans Affairs Healthcare System (VA HCS) population, pain is the most common symptom reported among patients in primary care, and $\sim 50 \%$ of these cases are treated by their primary care providers. ${ }^{1,2}$ In a 3month postdeployment survey sample of 2597 soldiers who had served in Afghanistan and Iraq, 44\% reported pain lasting for more than 3 months, half of whom reported pain for 1 year or more, and $15.1 \%$ who reported long-term opioid use for their pain management. ${ }^{3}$ Although opioids are effective for decreasing pain among patients with cancer and in hospice care, habitual use of these drugs for chronic pain carries severe risks. ${ }^{1,4}$ The body eventually develops a tolerance to opioids, so the same amount of medication no longer has the desired effect. ${ }^{5}$ This, in turn, leads to addictive behaviors and increases mortality rates from opioid-related

Whole Health Department, Fargo VA Health Care System, Fargo, ND.

This material is the result of work supported with resources and the use of facilities at the Fargo VA Health Care System. The contents do not represent the views of the U.S. Department of Veterans Affairs or the United States Government. 
overdoses. For these reasons, complementary and integrative medicine continues to expand within the VA HCS to mitigate the opioid crisis and provide an alternative to pharmacologic pain-management options.

Battlefield Acupuncture (BFA) was first developed by Richard C. Niemtzow, MD, PhD, MPH, in 2001. ${ }^{6}$ BFA is a unique, 5-point auricular acupuncture procedure that can be taught easily and is currently used in many military and VA HCS facilities. BFA has been described as a simple, safe, fast-acting, and cost-effective alternative to opioid medications for managing chronic pain. ${ }^{7}$ During the BFA treatment, the clinician places up to 5 auricular semi-permanent (Aiguille Semi-Permanente ${ }^{\circledR}$; ASP $^{\circledR}$ ) sterile needles in each ear. As with traditional auricular acupuncture, the entire body is represented in the ear. By placing these ASP needles in the surface of the ear, the whole body can be affected. ${ }^{8}$ This technique can deliver significant attenuation of pain in just a few minutes by stimulating the central nervous system and involving the hypothalamus, thalamus, cingulate gyrus, and cerebral cortex structures, which are thought to inhibit the neurotransmitters and inflammatory makers and release endogenous opioids. ${ }^{9}$ Studies using functional magnetic resonance imaging and positive electron tomography show that areas of the brain relating to pain sensation are inhibited when auricular acupuncture needles are in place. ${ }^{10,11}$ Therefore, BFA could be an effective nonpharmaceutical approach to pain management that could decrease the use of long-term opioids.

The Fargo VA HCS (Fargo, ND) has been offering acupuncture for pain management since December 2017, and now has 1 licensed acupuncturist on staff plus a medical doctor who offers BFA services in a group clinical setting. This retrospective cohort study expanded on current research by analyzing the immediate and long-term effects of BFA on pain intervention, also, by assessing the daily dose equivalence of patients who were on long-term prescription opioids 3 months prior to BFA and 6 months after BFA. This treatment group was compared to a randomly selected control group of veterans who were on long-term opioid pain contracts between December 2017 and November 2018 and who did not receive BFA. This article first reviews previous research on the effectiveness of BFA for pain management in a variety of settings and acupuncture treatments compared with pharmaceutical pain interventions. Then, there is an analysis of the Fargo VA HCS BFA group clinic's overall effectiveness for decreasing pain and opioid use in that veteran population and a cost analysis of implementing BFA treatments in a group clinical setting.

\section{Literature Review of Previous Research}

A literature search was conducted using the databases PubMed, Cochrane Database of Systematic Reviews, ClinicalKey, PsychInfo, the VA Office of Research and Devel- opment, and OvidMD. The keywords used were battlefield acupuncture, auricular acupuncture, BFA, battlefield acupuncture Veterans, opioid, and pain interventions. Articles reviewed were limited to hospital settings, acupuncture for pain management, and published within the last 10 years. Articles were selected based on the relevancy of the information the contained. This yielded a total of 8 articles selected: 1 meta-analysis of BFA for pain interventions in an emergency-room (ER) setting; 2 of BFA for pain management in a VA hospital setting; 2 on the guidelines and efficacy of long-term opioids, and 3 comparisons of the effectiveness of BFA for pain with pharmaceutical pain interventions.

\section{BFA Effectiveness for Pain Intervention}

Research on BFA for general pain management is still in its infancy. The majority of the current research on BFA for pain intervention in a clinical setting has been conducted by the VA or the U.S. military. The first meta-analysis on BFA - either as a stand-alone treatment or coordinated with other methods in an ER setting-was performed by the United Air Force Acupuncture and Integrative Medicine Center in 2017. ${ }^{12}$ The researchers there analyzed 6 randomized controlled trials (RCTs) ${ }^{7,13-17}$ and 2 observational studies ${ }^{18,19}$ and found significant results in decreased post treatment pain in all reviewed articles by an average of 2.5 points on a numeric rating scale (NRS) with no significant adverse effects and overall improved patient satisfaction.

Another cross-sectional study at the West Haven, CT, VA Medical Center was conducted over a 1-year period, where researchers analyzed 284 veterans with pain who received BFA in either an individual or group clinical setting. ${ }^{8}$ The researchers reported an immediate decrease in self-reported pain in $82 \%$ of patients, with no change in $9.7 \%$ of the patients, and an increase in pain in $8.3 \%$ patients. The researchers also reported that a decrease in the pain was common in both the group and individual settings, and the effectiveness of BFA to reduce pain remained consistent with ongoing use (once a week).

Finally, in an effort to increase the quality of life $(\mathrm{QoL})$ in the residents in the Community Living Center (CLC) at a VA center in Phoenix, AZ, researchers analyzed a new offering of BFA to the CLC residents. The researchers found that the patients' average pain score decreased by an average of 4.4 points after treatment. The researchers also reported that, in $85 \%$ of the patients, pain relief lasted for a few days and 4 of their 29 patients were evaluated and weaned off prescription opioids. ${ }^{20}$

Overall, studies showed that acupuncture is an effective treatment method in decreasing pain, with an average of 2.4 points reduction on the NRS immediately post-treatment. As a stand-alone treatment, these studies suggest that BFA can be significant for lowering pain immediately after treatment and this effect could be maintained with weekly treatments. ${ }^{8,19,20}$ However, when comparing BFA to other 
interventions, ${ }^{15-18}$ BFA effectiveness could depend more on the patients and the types of pain. It was suggested that BFA be used in coordination with standard medical care. Significant study bias and heterogeneity were found in several studies. ${ }^{72-19}$ Limitations of these studies are the small populations, uncontrolled clinical trials, and no blinding of patients or providers. In addition, previous research on the topic was quite limited and many researchers could have overestimated their results. In the future, there is a need for more RCTs to compare BFA to standard medical and pharmaceutical treatments for chronic-pain management to address study bias. Many of the researchers did call for further investigation into the potential long-term benefits of weekly BFA treatments for managing chronic pain., ${ }^{73-19}$

\section{Opioids' Effectiveness for Chronic Pain}

Opioids are commonly prescribed for chronic pain, with $3 \%-4 \%$ of U.S. adults currently prescribed long-term opioid therapy. ${ }^{21}$ However, much controversy surrounds their effectiveness and safety with long-term use. A previous metaanalysis of 15 RCTs showed that $\sim 80 \%$ of the participants within the studies had at least 1 adverse event (AE). ${ }^{22}$ Some of the most common AEs were nausea, vomiting, constipation, respiratory depression, and physical dependence. With chronic use, higher doses of opioids are needed to have the same pain-relieving effects. This commonly leads to potentially deadly overdoses.

According to the CDC Guideline for Prescribing Opioids for Chronic Pain-United States for providers by the Centers for Disease Control and Prevention (CDC), a systematic review found that opioids were moderately effective for pain relief, but there was a high percentage study dropouts due to AE. There was also no evidence, when addressing clinical questions, on the effectiveness of long-term opioid therapy for pain and QoL at least 1 year after starting opioid therapy. The guidelines instruct providers to evaluate patients within 1-4 weeks of starting long-term opioid therapy, and at least every 3 months afterward. ${ }^{23}$ The researchers did not find any studies proving the effectiveness of opioids for pain intervention for anything $>1$ year. Concern over the national opioid epidemic has led primary-care providers to decrease the number and duration of opioids they prescribe. In 2017, more than 47,000 Americans died as a result of opioid overdose, and veterans are twice as likely to die from accidental overdose than the civilian population. ${ }^{24, *}$

The dangers associated with the prescribing of opioids for chronic pain have led the Veterans Health Administration to

*Centers for Disease Control and Prevention (CDC)/National Center for Health Statistics. Underlying Cause of Death 2017 on CDC WONDER Online Database, 2018. Data are from the Multiple Cause of Death Files, 2017, as compiled from data provided by the 57 vital statistics jurisdictions through the Vital Statistics Cooperative Program. Accessed at http://wonder.cdc.gov/ucdicd10.html on May 28, 2019. require patients who are prescribed long-term opioids to sign an opioid contract that must be renewed, along with a urine drug screen biannually and state prescription drug monitoring every 90 days in order for their prescriptions to be renewed continually.

\section{BFA Effects on Decreasing Prescription Opioids}

Integrative care has been suggested as a safer alternative to opioids with less/no adverse effects in the treatment of chronic pain and has been shown to be an effective nonpharmaceutical treatment for chronic pain. ${ }^{25}$ Previous research on BFA or auricular acupuncture on its potential for decreasing prescription opioids is minimal. Among the literature there was no RCT comparing the effects of BFA and opioid use as a primary outcome measure. In the previously mentioned meta-analysis, ${ }^{12}$ prescription medication usage for pain management post ER treatments was measured as a secondary outcome. There was one RCT showing that patients who received BFA had reduction in their use of nonsteroidal anti-inflammatory drugs for sore throats. ${ }^{17}$ There was also a small pilot study showing no difference in ER opioid use; however, after the patients were discharged, fewer analgesia prescriptions were given to patients who had received BFA. ${ }^{15}$ It can be concluded from these studies that nonpharmaceutical interventions coordinated with pain medication could be better than just pain medication alone.

\section{MATERIALS AND METHODS}

Approval for this study was given from the institutional review board of the University of South Dakota. Review of medical records consisted of using data available from the computerized patient record system (CPRS).

\section{NRS Prior and Post-Treatment}

The NRS is an 11-point scale, ranging from 0 to 10 (with 10 being the worst imaginable pain), that is used for patients to self-report their pain. The NRS has previously been shown to have high test-retest reliability observed in both literate and illiterate patients before and after treatments ( $r=0.96$ and 0.95 , respectively) and is now commonly used throughout medical facilities. ${ }^{26,27}$ At primary-care appointments, either a registered nurse or a physician documents each patient's NRS score in an appointment chart note. For the Fargo BFA group clinic, the NRS was given orally to the provider prior to treatment, and then immediately after. The provider then documented the NRS in the patient's chart. All pain scores pre- and post- treatment for the treatment group were reviewed through the CPRS. Differences between scores were analyzed further by analysis of variance (ANOVA) ${ }^{28}$ and a Student's $t$-test, ${ }^{29}$ using SPSS (Armonk, NY). 


\section{NRS 3 Months Prior and 6 Months Post-Treatment}

Patients included in the study were prescribed opioids for chronic pain and assigned mandatory opioid contracts. These contracts require that the patient meets (in person or over the telephone) with their primary providers every 90 days for drug monitoring; NRS assessment; and vitals, such as weight, blood pressure, etc. All NRS pain scores 3 months prior to the initial BFA treatment and 6 months posttreatment for the treatment group were reviewed through CPRS and documented. The control group had their NRS scores analyzed near the beginning of the study period (the date closest to December 2017) and 6 months post this date. Analysis of the differences between groups was conducted through ANOVA ${ }^{28}$ using SPSS statistical software.

\section{Daily Dosage of Opioids 3 Months Prior and 6 Months Post-Treatment}

The milligram morphine equivalent (MME) for daily drug dosage was calculated using the CDC and Centers for Medicare guidelines. MME is a value assigned to opioids to represent their potencies using the formula:

$$
\begin{aligned}
& \text { Strength/Unit } \times(\text { Number of Units/Days' Supply }) \\
& \times \text { MME Conversion Factor }=\text { MME } / \text { Day. }{ }^{30,31}
\end{aligned}
$$

For the treatment group, MME was calculated by averaging 3 months prior to the patients' initial BFA treatments and 6 months post-treatment (ranging from August 2017 to January 2019). For the control group, MME was calculated by averaging 3 months prior to the study period, and 6 months post the study period. Further tests between the groups were analyzed again with SPSS statistical software for $\chi$-Square and ANOVA statistical analysis $(\alpha=0.01)$.

\section{RESULTS}

This retrospective cohort study analyzed 144 veterans who had attended the Fargo VA BFA group clinic. Of those who attended the clinic, $24(16.7 \%)$ were found to be on opioid contracts for chronic-pain management during the study period and were not excluded. Veterans were excluded if they were not on opioid contracts during the study period, were prescribed opioids for an acute injury and not for chronic pain, had not met with primary-care providers every 90 days for assessment and contract renewal, did not have documentation of their NRS scores every 90 days, or had initial NRS scores $\leq 4$ prior to BFA treatment.

The control group was randomly selected using a random number generator ${ }^{32}$ from a list of all veterans within the Fargo area who were on opioid contracts for the time of the study and who had not attended the BFA group clinic. Of the 50 veterans randomly selected, 23 met the inclusion criteria (See Fig. 1 for a breakdown of group criteria).

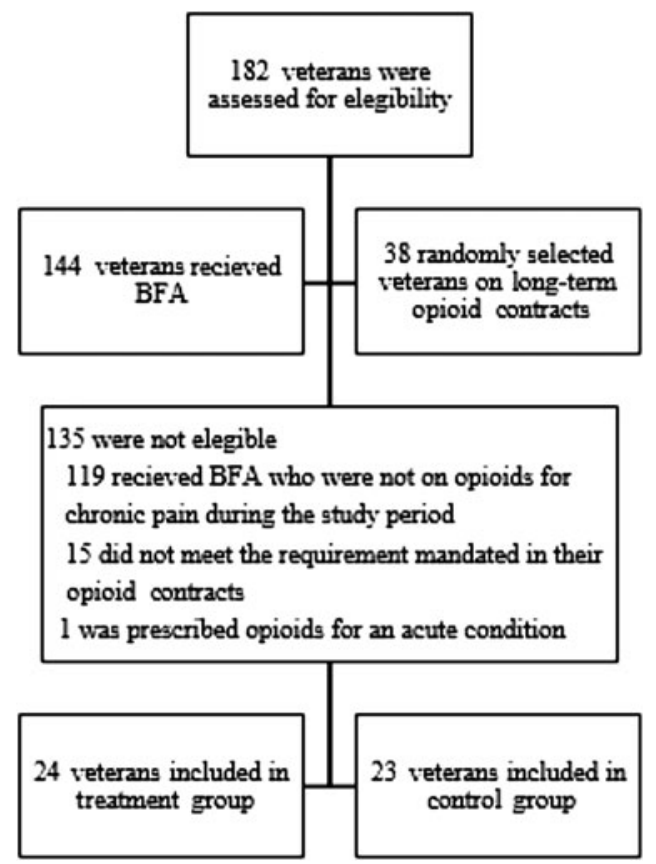

FIG. 1. CONsolidated Standards $O$ f Reporting Trials flowchart. BFA, Battlefield Acupuncture.

The treatment and control groups showed similar age distributions (mean [standard deviation; SD]: 61 [8.8], versus 65 [8.5] years) and race/ethnicity (white 23 [96\%] versus white 22 [96\%]). Pain diagnosis distribution showed some discrepancies between the treatment and control group (chronic back pain 14 [58\%] versus 12 [52\%], joint pain 6 [25\%] versus 7 [30\%], neck pain 4 [17\%] versus 8 [35\%], myofascial pain 5 [21\%] versus 2 [9\%], and chronic-pain syndrome in 5 [21\%]

Table 1. Demographic and Baseline Clinical Characteristics of Participants in Treatment and Control Groups

\begin{tabular}{lcc}
\hline & \multicolumn{2}{c}{$\#(\%)$ of participants } \\
\cline { 2 - 3 } & $\begin{array}{c}\text { BFA treatment } \\
\text { group } \\
\text { Characteristics }\end{array}$ & $\begin{array}{c}\text { Control } \\
\text { group } \\
(\mathrm{n}=24)\end{array}$ \\
\hline Age mean (SD), yr. & $61(8.8)$ & $65(8.5)$ \\
Male & $20(83.3)$ & $22(95.6)$ \\
Female & $4(16.7)$ & $1(0.04)$ \\
White race/ethnicity & $23(96)$ & $22(96)$ \\
Pain diagnosis ${ }^{\mathrm{a}}$ & $14(58.3)$ & $12(52.2)$ \\
Back & $6(25)$ & $7(30.4)$ \\
Joint & & $8(34.7)$ \\
Neck & $4(16.7)$ & $3(13.1)$ \\
Chronic pain syndrome $_{\text {Myofascial pain }}$ & $5(20.8)$ & $2(8.7)$ \\
\hline
\end{tabular}

${ }^{\mathrm{a}} 50 \%$ of the participants in treatment group and $43 \%$ of the control group had more than 1 area of diagnosed chronic pain.

${ }^{\mathrm{b} J o i n t}$ pain includes pain diagnosis in the shoulders, knees, elbows, hips, feet, and hands.

BFA, Battlefield Acupuncture; SD, standard deviation; yr., years. 
versus 3 [13\%]), respectively (Table 1). No participants in this study were diagnosed with chronic cancer pain.

\section{NRS and BFA Treatment}

There was a significant average decrease in the NRS scores immediately post BFA treatment of 1.3 points $(P<0.01)$. The NRS scores 3 months prior to BFA and 6 months after BFA showed no statistically significant difference in relation to each other $(P>0.01$, mean difference $=-0.2$ ). In the control group, the average NRS score remained at a level of 7 for the duration of the study period with no significant change $(P>0.01)$. Analysis by a Student's $t$-test showed that, between the treatment and control groups, there were no significant differences during the 9 months of documented NRS scores $(P=0.15)$.

\section{BFA and Opioids}

After calculating the average MME for the treatment group 3 months prior to the initial BFA treatment and 6 months post the initial treatment, ANOVA and a Student's $t$-test were used for differences. The total average MME of opioids in the treatment group was 31.0 and 45.05 in the control group. In the treatment group, 3 of the 24 veterans $(12.5 \%)$ had decreased final dosage of MME of opioids. This was not significant between the average of the first 3 months and the 6 months following $(P=0.28)$. In the control group, 4 of the 23 veterans $(17.4 \%)$ had decreases in their MME. Over the 9 months of evaluation, the veterans' average MME increased by 3.9 in the treatment group and by 8.7 in the control group.

\section{DISCUSSION}

\section{NRS and BFA Treatment}

BFA is growing in popularity as an effective nonpharmaceutical pain-alleviating method. The verbal responses of the veterans pre- and post-treatment were very positive. Anecdotally, clinical documentation indicated that the veterans: "Feel much better with BFA" and "Is even going off methadone and has better mental clarity." Overall, the numbers of the Fargo VA population were similar to larger populations in previous studies ${ }^{7,8,12-20}$ with a significant average decrease of 1.3 points on the $0-10$ NRS $(P<0.01)$. See Table 2 .
As a retrospective study, no causal characteristics were found, and it was not possible not account for variabilities such as if a veteran had started other nonpharmaceutical pain interventions such as full-body acupuncture, massage, or chiropractic therapy for chronic pain within the VA or in the community. An analysis of the differences of NRS 3 months prior and 6 months post-treatment showed that there to be no significant differences in pain scores $(P>0.01)$. This was not surprising, as it was shown in other studies that auricular acupuncture/BFA are most effective if performed as consecutive weekly treatments. ${ }^{8,20}$ Research has not found that there is a limit to the amount of treatments a patient receives for full-body or auricular acupuncture. ${ }^{33}$ As the pain reducing effects of BFA are reported to last for up to 1 week, veterans come back regularly, weekly for regular treatments with no end or limit to the amount of times veterans can come for maintenance care. ${ }^{8,20,33}$

\section{BFA and Opioids}

After analyzing the average MME 3 months prior, and 6 months post-treatment in the treatment group and control group, there was no a significant differences in the amount of medication used for chronic pain. This could be due to the physical dependence that comes with long-term use of opioid prescriptions. A higher percentage of patients in the control group (17.4\%, compared to $12.5 \%$ in the treatment group) had reductions in their average opioid use for chronic pain. This could be, in part, due to the fact that the treatment group had a lower average dose of average MME for opioids, compared to the control group (31.0 versus 45.05, respectively). Further research using a multisite RCT approach with a preliminary power-analysis and a larger population is needed to show any significant difference. No research was found that had been conducted to prove the effectiveness of long-term opioid treatments for decreasing pain, and that opioids were more effective in the span of $\leq 6$ weeks for patients to report decreases in their pain. ${ }^{22}$ Patients who were on opioid-contracts analyzed in this study had been on opioids for $>6$ weeks. These opioids would no longer have had the effectiveness of initial opioid treatments and the patients were at an increased risk of opioid dependence and overdosing. ${ }^{23}$

\section{Cost Analysis of BFA Versus Opioids}

The cost of opioid treatments varies widely due to many factors. For example, there are a variety of opioid medications

Table 2. NRS in Patients Pre- and Post-BFA Treatment

\begin{tabular}{lcccc}
\hline Factors & Fargo VA BFA clinic & Goertz et al., 2006 & Moss \& Crawford, 2015 & Federman et al., 2018 $^{8}$ \\
\hline $\begin{array}{l}\text { Mean pain score change } \\
\text { difference after intervention }\end{array}$ & 1.3 & 2.9 & 4.5 & 3.35 \\
NRS pretreatment & 5.2 (SD: 1.89) & 6.98 (SD: 1.68) & 6.4 (SD: 1.86) & 5.69 (SD: 2.06) \\
NRS post-treatment & 3.9 (SD: 2.07) & 4.08 (SD: 1.99$)$ & 1.9 (SD: 1.09$)$ & 2.34 (SD: 1.96$)$ \\
$p$-Value & 0.003 & 0.005 & 0.0001 & 0.0001 \\
\hline
\end{tabular}

NRS, numeric rating scale; BFA, Battlefield Acupuncture; VA, Veterans Administration; SD, standard deviation. 
that have a wide range of pricing. With factoring in the cost of care and medical support needed for patients who are prescribed long-term opioids, the U.S. Department of Defense reports that cost estimates for patients who are in certified opioid-treatment programs average anywhere from $\$ 115.00$ / week to $\$ 294.13 /$ week, depending on the types of prescriptions and the amount of monitoring needed. ${ }^{34}$

BFA can be administered in a group clinical setting and takes roughly 10 minutes per treatment. As previously mentioned, the provider places up to 5 ASP ear needles in each ear during BFA. After each needle is placed, the veteran is asked to walk around the room before placing the next needle. A box of 200 ASP ear needles costs $\$ 81.00$ and have been shown to stay in place for an average of 7 days before falling out. ${ }^{35}$ To break that down, 200 needles would be used to treat 20 veterans for 1 week of pain relief, or $\$ 4.05$ per veteran, per week, or $\$ 210.60$ for a year of weekly treatments. The clinic would also need the provider for the duration of the clinic's hours of operation and administrative personnel to make the appointments. The Fargo BFA group clinic operated for 3 hours at a time with 15-minute appointment slots that were often overbooked ( 2 or more veterans in 1 appointment slot). As a total, the licensed physician, needles, and administration would cost roughly \$240 per week for 13 -hour group clinic treating 15-20 veterans per week (roughly $\$ 14 /$ week/veteran). However, this price would vary by state and by the types of providers administering the BFA. For example, if the state allows registered nurses to administer BFA after attending training, the cost of implementation would be reduced.

\section{Needs for Future Research}

Research is ongoing on the effect of BFA on immediate and long-term pain relief. Although the current retrospective cohort did not show significant evidence that this immediate pain relief can influence the amount of opioids used, future research is needed with RCTs in a larger population that can account for more variables. In addition, future research is needed on the number of treatments needed with BFA for effective pain management and on how often patients should return for treatments.

\section{Study Limitations}

This study was a retrospective cohort analysis, using a quasiexperimental design (comparing existing groups), therefore, it could not show causal relationships between the treatment of BFA on the potential decrease over time of opioid pain management. This kind of study did not allow for randomized assignment of participants to various research conditions for the treatment group. No blinding of the treatment group was used, and bias between the provider and the patients might have occurred. As with many retrospective cohort studies, some information was lost to follow-up and potential confounding factors and bias were viable. ${ }^{36}$

\section{CONCLUSIONS}

The amount of research into the practice and methods of BFA, compared to other modalities, is limited. BFA is an effective nonpharmaceutical pain intervention method that can be paired with other forms of pain interventions or used as a standalone treatment in either an individual or group setting. When treating a patient with chronic pain, allowing for more than one method of treatment can be most effective, as each patient's pain is subjective. These preliminary results showed that BFA performed in a group setting was an effective pain-intervention method and was safe to use on a weekly basis for ongoing pain management for veterans. Possible future directions for implementing BFA could be used as an optional stand-alone pain intervention method, in coordination with a 90-day contract to manage pain without adding complications with other medications, or prior to surgical procedures to potentially lower the amount of pain medication needed postoperatively.

With the increasing amount of severe complications that come from chronic use of opioids for pain management, using an option such as BFA has the potential to help decrease the dose of opioids a patient needs to receive. Future research is needed to understand how the different types of pain, comorbidities, access to healthcare, and other nonpharmaceutical options for pain would interact with BFA as a treatment option.

\section{AUTHOR DISCLOSURE STATEMENT}

No financial conflicts of interest exist.

\section{FUNDING INFORMATION}

No funding was received for this article.

\section{REFERENCES}

1. Volkow N, Benveniste H, McLellan AT. Use and misuse of opioids in chronic pain. Annu Rev Med. 2018;69(1):451-465.

2. Andersson GB. Epidemiological features of chronic low-back pain. Lancet. 1999;354(9178):581-585

3. Toblin RL, Quartana PJ, Riviere LA, Walper KC, Hoge CW. Chronic pain and opioid use in US soldiers after combat deployment. JAMA Intern Med. 2014;174(8):1400-1401.

4. Gomes T, Mamdani MM, Dhalla IA, Paterson JM, Juurlink DN. Opioid dose and drug-related mortality in patients with nonmalignant pain. Arch Intern Med. 2011;171(7):686-689.

5. Martell BA, O'Connor PG, Kerns RD, Becker WC, Morales KH, Kosten TR, Fiellin DA. Systematic review: Opioid treatment for chronic back pain. Prevalence, efficacy, and association with addiction. Ann Intern Med. 2007;146(2):116-127.

6. Niemtzow RC. Battlefield Acupuncture. Med Acupunct. 2007; 19(4):225-228.

7. Goertz CM, Niemtzow RC, Burns SM, Burns SM, Fritts MJ, Crawford CC, Jonas WB. Auricular acupuncture in the 
treatment of acute pain syndromes: A pilot study. Mil Med. 2006;171(10):1010-1014.

8. Federman DG, Zeliadt SB, Thomas ER, Carbone GF, Taylor SL. Battlefield Acupuncture in the Veterans Health Administration: Effectiveness in individual and group settings for pain and pain comorbidities. Med Acupunct. 2018;30(5):273-278.

9. Clement-Jones V, McLoughlin L, Tomlin S, Besser GM, Rees LH, Wen HL. Increased beta-endorphin but not met-enkephalin levels in human cerebrospinal fluid after acupuncture for recurrent pain. Lancet. 1980;2(8201):946-949.

10. Romoli M, Allais G, Airola G, et al. Ear acupuncture and fMRI: A pilot study for assessing the specificity of auricular points. Neurol Sci. 2014;35(suppl1):189-193.

11. Cho ZH, Fallon J, Wong EK. Neuro-Acupuncture, vol I: Basic Neuroscience. Los Angeles: Q-Puncture Inc.; 2001.

12. Jan AL, Aldridge ES, Rogers IR, Visser EJ, Bulsara MK, Niemtzow RC. Does ear acupuncture have a role for pain relief in the emergency setting? A systematic review and meta-analysis. Med Acupunct. 2017;29(5):276-289.

13. Allais G, Romoli M, Rolando S, Airola G. Castagnoli Gabellari I, Allais R, Benedetto C. Ear acupuncture in the treatment of migraine attacks: A randomized trial on the efficacy of appropriate versus inappropriate acupoints. Neurol Sci. 2011;32(suppl1):S173-S175.

14. Barker R, Kober A, Hoerauf K, Latzke D, Adel S, Kain ZN, Wang SM. Out-of-hospital auricular acupressure in elder patients with hip fracture: A randomized double-blinded trial. Acad Emerg Med. 2006;13(1):19-23.

15. Fox LM, Danesh H, Murakami M, et al. Randomized trial of acupuncture versus standard therapy to treat low back pain in the emergency department [abstr]. Ann Emerg Med. 2016;68(4):S81.

16. Gu X. Clinical study on analgesia for biliary colic with ear acupuncture at point Erzhong. Am J Acupunct. 1993;21(3):237-239.

17. Moss DA, Crawford P. Ear acupuncture for acute sore throat in patients unable to take non-steroidal anti-inflammatory agents (NSAIDs). J Am Board Fam Med. 2015;28(6):697-705.

18. Burns S, York A, Niemtzow RC, Garner BK, Steele N, Walter JAG. Moving acupuncture to the front line of military medical care: A feasibility study. Med Acupunct. 2013;25(1):48-54.

19. Graff DM, McDonald MJ. Auricular acupuncture for the treatment of pediatric migraines in the emergency department. Pediatr Emerg Care. 2018;34(4):258-262.

20. Shao X, Byers M, et al. Battlefield Acupuncture (BFA) for pain management in a VA community living and rehabilitation center. JAMDA. 2018;19(3):B14.

21. Boudreau D, Von Korff M, Rutter CM, et al. Trends in longterm opioid therapy for chronic non-cancer pain. Pharmacoepidemiol Drug Saf. 2009;18(12):1166-1175.

22. Kalso E, Edwards JE, Moore AR, McQuay HJ. Opioids in chronic non-cancer pain: Systematic review of efficacy and safety. Pain. 2004;112(3):372-380.

23. Dowell D, Haegerich TM, Chou R. CDC Guideline for Prescribing Opioids for Chronic Pain-United States. JAMA. 2016;315(15):1624-1645.

24. Talbott Recovery. Opioid Addiction: One More Way We're Failing Our Veterans. September 21, 2017. Online document at: https://talbottcampus.com/opioid-addiction-one-more-waywere-failing-our-veterans/ Accessed May 28, 2019.
25. Stanford Health Care. Management of Pain without Medications. Stanford Medical Center. Online document at: https:// stanfordhealthcare.org/medical-conditions/pain/pain/treatments/ non-pharmacological-pain-management.html. 2018. Accessed February 22, 2019.

26. Hawker GA, Mian S, Kendzerska T, French M. Measures of adult pain: Visual Analog Scale for Pain (VAS Pain), Numeric Rating Scale for Pain (NRS Pain), McGill Pain Questionnaire (MPQ), Short-Form McGill Pain Questionnaire (SFMPQ), Chronic Pain Grade Scale (CPGS), Short Form-36 Bodily Pain Scale (SF-36 BPS), and Measure of Intermittent and Constant Osteoarthritis Pain (ICOAP). Arthritis Care Res (Hoboken). 2011;63(suppl11):S240-S252.

27. McCormack HM, Horne DJ, Sheather S. Clinical applications of visual analogue scales: A critical review. Psychol Med. 1988;18(4):1007-1019.

28. Bewick V, Cheek L, Ball J. Statistics Review 9: One-Way Analysis of Variance. Critical Care. London. Online document at: www.ncbi.nlm.nih.gov/pmc/articles/PMC420045/ Accessed April 2, 2019.

29. Student. The probable error of a mean. Biometrika. 1908; $6(1) 1-25$

30. Opioid Oral Morphine Milligram Equivalent (MME) Conversion Factors. Centers for Medicare \& Medicaid Services; 2017. Online document at: www.cms.gov/Medicare/PrescriptionDrug-Coverage/PrescriptionDrugCovContra/Downloads/OpioidMorphine-EQ-Conversion-Factors-Aug-2017.pdf Accessed September 29, 2019.

31. Centers for Disease Control and Prevention. Calculating Total Daily Dose of Opioids for Safer Dosage. 2017. Online document at: www.cdc.gov/drugoverdose/pdf/calculating_total_daily_dosea.pdf Accessed May 22, 2019.

32. Haahr M. RANDOM.ORG: True Random Number Service. 2019. Online document at: www.random.org Accessed July $15,2019$.

33. Hempel S, Shekelle PG, Taylor SL, et al. Evidence Map of Acupuncture: Evidence-Based Synthesis Program. Washington, DC: Department of Veterans Affairs; 2014.

34. U.S. Department of Defense, Office of the Secretary. TRICARE; Mental Health and Substance Use Disorder Treatment. Federal Register. Online document at: www.federalregister.gov/ documents/2016/09/02/2016-21125/tricare-mental-health-andsubstance-use-disorder-treatment Accessed May 28, 2019.

35. Umeh B. Ear acupuncture using semi-permanent needles: Acceptability, prospects and problems in Nigeria. Am J Chin Med. 1988;16(1-2):67-70.

36. Euser AM, Zoccali C, Jager KJ, Dekker FW. Cohort studies: Prospective versus retrospective. Nephron Clin Pract. 2009; 113(3):c214-c217.

Address correspondence to: Anna Denee Montgomery, BS Whole Health Department Fargo VA Health Care System 2101 Elm Street Fargo, ND 58102-2417 E-mail: anna.montgomery2@va.gov 